\title{
A General Framework for Time Series Forecasting Model Using Autoregressive Integrated Moving Average-ARIMA and Transfer Functions
}

\author{
G. Solomon Osho \\ Correspondence: Associate Professor of Economics, Department of Management and Marketing, Prairie View A\&M \\ University, USA.
}

Received: October 23, 2017 Accepted: September 4, 2019 Online Published: September 25, 2019

doi:10.5539/ijsp.v8n6p23 URL: https://doi.org/10.5539/ijsp.v8n6p23

\begin{abstract}
Major current econometric stochastic series forecast research are established on the failure of the scholastic process tests to differentiate between finite and stationary alternative samples of the unit root hypothesis results. The importance of forecast evaluation allows researchers to reasonably monitor and improve forecast performance. While a structured improved forecast framework have often been suggested as one possible alternative, an extended the multivariate model which incorporate distributed-lag period for independent variable gives a unique advantage over the traditional distributed-lag model and the mathematical formulation does essentially guarantee that predicated equation irrespective of the values of the predictor variables. Hence, the primary objective is mainly to determine the likelihood of autoregressive integrated moving average (ARIMA) method for practicable process choice used for predicting key economic variables for a set of market data. Once the process has been known, parameters have been obtained, and the adequacy of the model has been determined, forecasts can be checked for reliability.
\end{abstract}

Keywords: econometric forecasting, time series, ARIMA and transfer model

\section{Introduction}

The general knowledge in the fields of economics and finance reveals that the time of complete doubt is the optimal time to buy, and while the time of maximum confidence is the optimal time to sell. Therefore, forecasts are of great significance and generally deployed in economics and trade; and sound forecasts technically lead to clear decisions. The significance of forecast evaluation enables users to systemically monitor and improve forecast performance. Economists have proposed various models for analyzing price formation in the world market through a forecasting framework. In general, the literature on forecasting offers two main approaches. These approaches are called causal forecasting and econometric forecasting models.

First, causal forecasting model uses historical data to estimate the relationship between the variable to be forecasted that is, the response variable and other variables such as independent variables or explanatory variables. It is built on a known correlation between the predicted and other exogenous variables. Econometric forecasting appears in number of different forms: first, a regression statistical technique for estimating a relationship between endogenous variable to one or more set of exogenous variables that are believed to influence the dependent variable. Second, an input-output matrix that defines the inflows from one segment of the economy to another, and therefore forecast the inputs needed to generate outputs in another economy segment. Third, simulation technique offers valuable information on plausible changes to a target objective function due to changing sensitivities of exogenous variables (DeJong and Dave, 2007).

Second, the econometric forecasting technique is mainly aimed to provide a more process for producing forecast values of a trade system grounded on current well established trade relationships that are designed to integrate a range of economic associations appropriate for long-term forecasts (Clements and Hendry, 2002). Furthermore, the econometric time series process can utilize data from the trade accounting matrix modeling process to deliver a more robust mechanism for making long-term forecasts of vital trade sectors and their interrelatedness.

Also, commodity market has a lot of forecasting uncertainty basically due to several significant variables such as exchange rates and climatic conditions that are often difficult to forecast (Labys, 1999). Cooper and Nakanishi (1988) established an extended the multivariate model which incorporate two instead of one exogenous variables in the with lag period. The advantage is that it does inevitably guarantee that predicated equation irrespective of the values of the exploratory variables. Hence, the primary objective is mainly to provide a general framework for determining the 
likelihood of ARIMA as a method for a practicable process choice for predicting key market variables for a set of economic data. Once the process has been known, parameters have been obtained, and the adequacy of the model has been determined, forecasts can be checked for reliability.

\section{Prior Studies}

Enders (2003) explained that forecast users must constantly and properly incorporate all pertinent information available in building forecasting models. Their results showed that forecast relevancy must also be checked by evaluating if the forecast errors are predictable. Furthermore, the data and technique must be evaluated and checked for suitability and presented testing procedures for forecast relevancy. Contrastingly, results of prior research, the test findings showed that forecast value for price seem to be rational. Nevertheless, this study does not take into account that the joint tests of specification and forecast rationality of variables are often essential, since market and trade flows are not static. Hence, a multivariate forecast should be considered in order to show a strong market relationship. Tellis, 1989 noted that finite-lagged model equations are regularly applied in research studies to developing market share models, where market share was generally quantified as the endogenous lagged share and relative price as exogenous variable. Therefore, according to economic theory, the addition of this crucial variable can increase the forecasting accuracy of the model.

Samarendu, Meyers and Danell (1999) examined price fluctuation and association in the global wheat market between 1981-1993 by means of a cointegration and error correction method. The findings show confirmation that the U.S., European Union, Argentina and Australia respond to Canadian pricing decisions. Additional findings indicate that Canada does not respond to price changes relative to Australia since price series were noted to be difference stationary (d1) and cointegrated (I). However, their research did not account for nonstationarity in the price series and as a result, co-integration could be misspecified because other essential economic variables like gross domestic product, interest rates and others were not included in the model.

A vector autoregression method was used to assess dynamic interactions in the international prices of wheat with cost of transporting goods and exchange rates as exogenous factors. Variance decomposition of forecast errors and transfer functions were used to inspect fluctuation in prices for selected global wheat markets. Findings suggested important continuous model interactions in wheat prices globally due to exchange rates and transportation costs. However, instead of using price ratios, their model presumed exchange rates. It is often suggested that exogeneity of price ratios and gross domestic product could be used instead of exchange rates because they can influence trade flows and price expectation (Goodwin and Schroeder, 1999).

Diebold (2007) concluded that trend, differences/levels unit root and cointegrated models with similar time series processes often generate very dissimilar forecast values. Ultimately, how to determining which time series process to apply is extremely significant to forecasters. Dickey, Bell, and Miller (1986) therefore suggested that instead of deploying a single time series process by default, it is critical to first apply a unit root test for stationarity as a diagnostic instrument for proper model selection decision. In effect, the primary incentive for tests of stationarity was specifically to aid determine whether to apply a time series process in differences or levels.

Major current econometric forecast models research are based on the failure of the scholastic process tests to differentiate between finite and stationary alternative samples of the unit root hypothesis results. The importance of forecast evaluation allows researchers to reasonably monitor and improve forecast performance. It is well emphasized that the precision of forecast values can be increased by using a time series process in differences instead of process in levels when the root is stationary. In the end, the main of goal for forecasting is not whether stationarity pretests the selection of the true model, but rather if it selects the model that produces dominance forecast values. Remarkably very less information is known on the robustness and efficiency of stationarity tests for the goal.

\section{The Theoretical Framework}

A univariate stochastic $\left(y_{t}\right)$ is known to be stable and in stationarity when the expected value of the series is independent of time and finite. All periods of the variable have the same finite mean:

$$
E\left(y_{t}\right)=E\left(y_{t-s}\right)=\mu_{x} .
$$

A variability measured by variance of the univariate stochastic is finite and over time is independent. Thus:

$$
E\left(y_{t}^{2}\right)=E\left(y_{t-s}^{2}\right)=\sigma_{y}^{2} .
$$

where $\mu_{y}, \sigma_{y}^{2}, \gamma_{s}$ denotes constant and stationary. If the three conditions hold, this series sequences shows weak stationarity. If the probability distribution $\mathrm{P}\left(y_{1}, y_{2}, \ldots, y_{t}\right)$ is also stationary, then the process is completely stationary and if the process changes overtime, then it is a non-stationary time series (Granger, 1986).

Most times series of economic variable exhibits non-stationary in level (variable before differencing). Such time series 
are subjected to detrending procedures to make them stationary before proceeding with further analysis. If stationarity is achieved after fitting a time trend, the variable is said to be trending stationary. The trend stationary process arises because of the effect of a deterministic trend. The second approach is to take the first difference of the stochastic process of interest and use it as a detrend stationary. If stationarity is achieved after differencing, the variable is said to be difference stationary. An advantage of the second approach is that if the series are in log levels, the first differences are approximately the percentage change over the previous period (Granger, 1986).

The econometric studies on unit root took off after the publications in 1982 argued that several economic forecasting exhibit stationarity and that is important for the analysis of economic policies and the difference unit root series known to be integrated (Nelson and Plosser, 1982). A more general standard inference technique cannot be applied to regressions with integrated explanatory variable. Hence, it is imperative to test for stationarity prior to using a regression and the strict procedure for checking a stationarity of a process is to exam for a unit root.

\section{Model Selection}

Generally, a model selection procedure for a time series forecast must consider among several factors first, what is the purpose of the forecast, what data period available, easiness of applicability, operational cost, and the degree of accuracy required. Makridakis (1986) suggested that the two most important model selection criteria are simplicity of use and forecast accuracy. However, Anandalingan and Chen (1989) stressed that forecast accuracy is single most critical criterion for selection of a forecasting model. Conversely, Dalrymple, (1987) specified that there is no distinctive process that is always accurate at all times but there is important proof to recommend that simple forecast model is usually preferred.

In addition, Box and Jenkins (1976) noted when a stochastic time series model is fully studied, it is possible to define and empirically fit it precisely with a generalized mathematical expression. First, a proper time series model is selected and tests are conducted to specify an approximate set of processes by identification of their classes. Second, a well-studied process is fitted by using available data. This is carried out in order to obtain parameter estimates. Third, diagnostic checks are conducted to determine any deficiency fit and If none, the estimated time series is then attempted for real data forecasting. Conversely, if any unsuitability is detected, the repetitive phase of identifying, estimating, and assumptions checking is reiterated pending when an appropriate time series forecast process is obtained (Diebold, 2007).

The numerical forecast measures of the stochastic process are estimated using the degree of the time series that defines that process. The principle of parsimony which is widely used by forecasters states that a time series process must sufficiently characterize the information with as limited coefficients as possible. Therefore, the central mechanism is focused on attaining a proper probabilistic process for predicting numerical estimates of the time series. Probabilistic process model can be a forecasting model representing the behavior of a phenomenon in a probabilistic fashion. Stochastic model cannot be predicted based solely upon their historical behavior (Granger and Newbold, 1986). The stochastic time series processes can be inferred as explanations of physical models possessing the right overall features but without precise physical models that are not fitted to empirical data.

The theoretical underpinnings used by Box and Jenkins (1976) are quite sophisticated, to the extent that nonspecialists utilize to get a comprehensive validity of the methodology. A study conducted in 1971 suggested that the original procedure of Box and Jenkins for seasonal and non-seasonal forecasting is better than the early versions of the Box-Jenkins procedure (Harrison \& Scott 1971). In additional, Box-Jenkins (1976) effectively collected significant data in a robust routine that required knowledge of univariate time series process. The basis of this approach is presented below and reflects three phases of identifying, estimating and testing phase, and application phases (Figure 1) (Gershenfeld and Weigend, 1994). The primary steps in generating the Box-Jenkins Process: Identification of ARIMA

- Parameters Estimation using OLS/GLS

- Diagnostic

- Checking for model adequacy

- Applicability

If the initial model is insufficient for used, another ARIMA models is considered by repeating the above procedure until a "satisfactory" model is found (Harrison and Scott, 1971). By assuming that $\mathrm{n}$ successive serial observations $y_{1}, y_{2}$, $y_{3}, \ldots$, are given for a model proper determination. Let the value for the univariate with equidistant time:

$$
t, t-1, t-2, \ldots \text { by } y_{t}, y_{t-1}, y_{t-2}, \ldots
$$

B denotes the backward regressive function such that 


$$
\begin{gathered}
B y_{t}=y_{t-1} \\
(1-B) y_{t}=y_{t}-y_{t-1}=\nabla y_{t}
\end{gathered}
$$

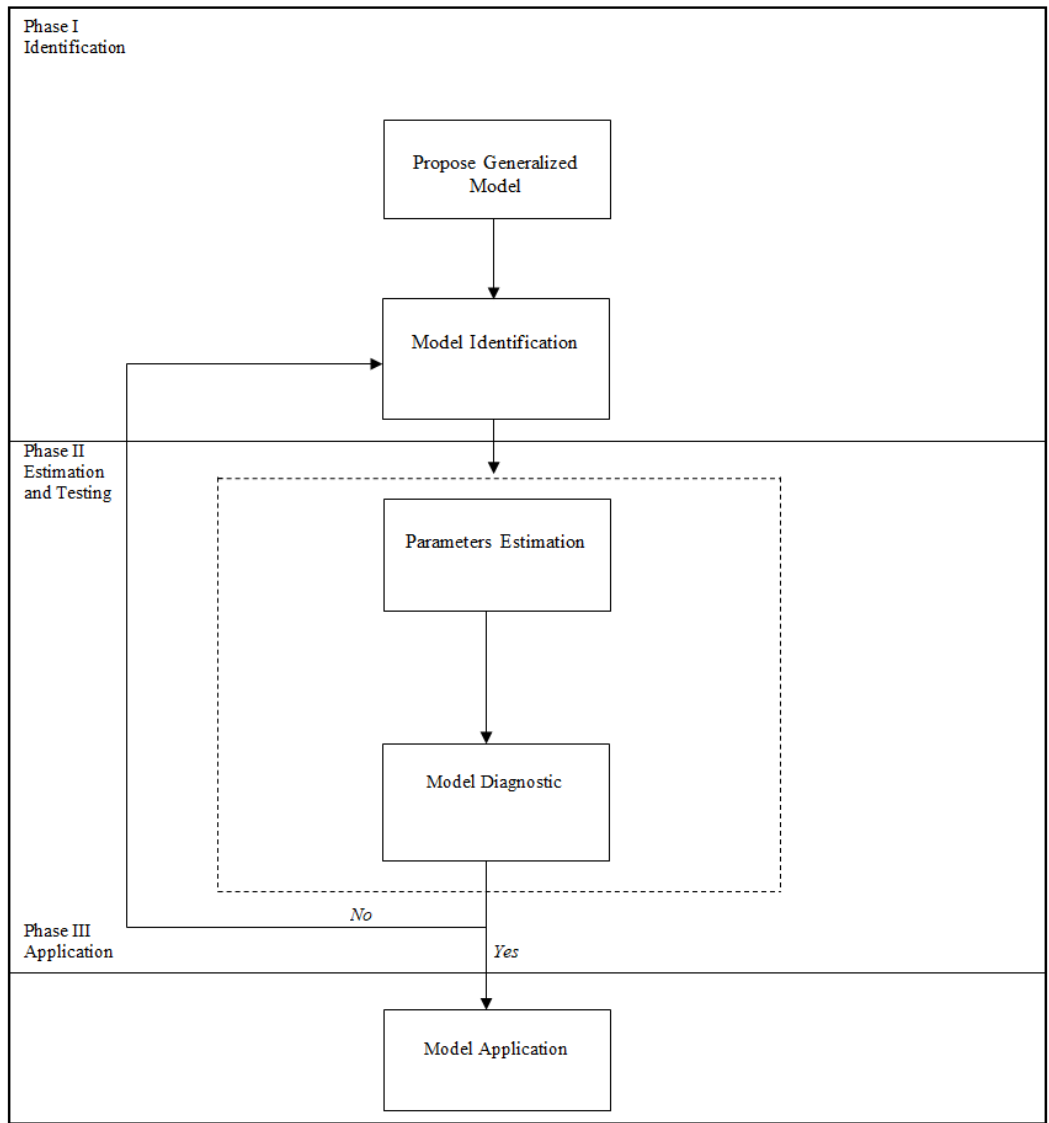

Figure 1. Schematic for Box-Jenkins Method

The suitable model for univariate non-stationary processes of the type that occurs in many trade models with ARIMA ( $p$, $d, q$ ) model:

$$
\begin{gathered}
\phi_{p}(B) \nabla^{d} y_{t}=\theta_{q}(B) a_{t} \\
\phi_{p}(B) \nabla^{d} y_{t}=C+\theta_{q}(B) a_{t}
\end{gathered}
$$

This results into an AR operator polynomial of the $p$-order

$$
\phi_{p}(B)=1-\phi_{1} B-\ldots-\phi_{p} B^{p}
$$

and MA operator polynomial of the $q$-order:

$$
\theta_{q}(B)=1-\theta_{1} B-\ldots-\theta_{q} B^{q}
$$

The aim for bring a finite autoregressive $\phi_{p}(B)$ and a converging moving average $\theta_{q}(B)$ together is that a finite MA is comparable to an infinite AR univariate sequence such as including they are parsimony in that particular order and form. The $\nabla^{d}$ with $d$-order differencing operator introduces factor termed as homogeneous non-stationarity with $a_{t}, a_{t-1}$, $a_{t-2}, \ldots$ as random variables identical, independent, and normally distributed (Granger, 1989). Assume the time series process for a univariate has been tentatively identified and specified as (

$$
\phi(B) \nabla^{d} y_{t}=\theta(B) a_{t} .
$$


Thus, for $y_{t}$ and any given $\phi(B)$ and $\theta(B)$ :

$$
a_{t}=\frac{\phi(B)}{\theta(B)}(1-B)^{d} y_{t}
$$

The log likelihood of any given $\phi(B)$ and $\theta(B)$ is strictly converged to linear function with sum of the squares deviation residual:

$$
S(\phi, \theta)=\sum_{t=1}^{n} a_{t}^{2}
$$

The following two basic procedures for checking the fitness of a time series model: examining the residual $a_{t} \mathrm{~s}$ and conducting the degree of overfitting. With this approach, if the time series process is satisfactory and degree of fitted of observations is adequate, then the parameter values $(\hat{\phi}, \hat{\theta})$ are appropriately close to $(\phi, \theta)$ and therefore, the residuals $a_{t}(\phi, \theta)$ are uncorrelated. However, if there is an exact need to further identified the time process, it will be checked, a more complex check procedure is used to differentiate the fitness of the previous specified model.

Furthermore, an extensive full choice of suitable practicality of the model is attained when a prospective conversion is permissible. Therefore $y_{t}^{(\lambda)}$ is replaced with $y_{t}$ where $y_{t}^{(\lambda)}$ is a nonlinear conversion of parameters $\lambda$. An appropriate transformation is often recommended by the simulated position and predicated from the series. For instance, when $y_{t}$ is marginally increasing and percentage oscillation showed a rather stability in the absolute oscillation, it is reasonable to evaluate the $\log$ form of $y_{t}$ (Box, Jenkins and Rinsel, 1994).

Let assume a time series process in the form $\phi_{p}(B) \nabla^{d} y_{t}=C+\theta_{q}(B) a_{t}$ has been identified and specified. A minimum MSE value $\hat{y}_{t}(l)$ for $y_{t+l}(l \geq 1)$, can be generated. In general, this provides a polynomial function of present and prior observations $y_{t}, y_{t-1}, \ldots$ with coefficients in a linear form of present and prior unpredictable changes $a_{t}, a_{t-1}, \ldots$. In theory the time series process are typically easily obtained by fitting a probabilistic function of the form:.

$$
\phi(B)=\theta(B)(1-B)^{d}=1-\phi_{1} B-\phi_{2} B^{2}-\ldots-\phi_{p+d} B^{p+d}
$$

After obtaining the probabilistic function, the model is fitted then applied using data to get the serial information to acquire the forecast values such as:

$$
y_{t+1}=\phi_{1} y_{t+l-1}+\ldots+\phi_{p+d} y_{t+1-p-d}-\theta_{1} a_{t+l-1}-\ldots-\theta_{q} a_{t+l-q}
$$

The backward shift operator is noted as $B$ is, $d$ is the degree of differencing involved, AR operator function of a $p$-order and MA operator function of a $q$-order.

A forecast at original $t$ at the initiating of a process $l$ is obtained by taking restricted likelihood at time $t$ of the equation. A $y_{t-j}(j=0,1,2, \ldots$,$) that previously succeeded at initial base t$ are left intact. This $y_{t+j}(j=1,2, \ldots)$ that has not succeeded is substituted by their estimates $\hat{y}_{t}(l)$ at initial base $t$. The $a_{t-j}(j=0,1,2, \ldots)$ that has succeeded is accessible from $y_{t-j}-\hat{y}_{t-j}$. The $a_{t+j}(j=1,2, \ldots)$ that have not succeeded are substituted by zero. This generate forecast $\hat{y}_{t}(l)$ completely for prior forecast value and present values of the series.

By applying this procedure, only previous values of a univariate variable are deployed to predict future values. Hence, commonly a model as described thus does not completely neglect the influence of the other related variables on past values. It does account for variation on past estimates of the univariate serial process partially allowing past values of the initial univariate series to predict themselves. Nevertheless, an improved result often an achieved via a transfer polynomial process which would definitely account for other related variables like inflation, exchange rate and other key economic variables.

Another application of stochastic process method is consistent with conditions that the predicted errors of the univariate forecasts are associated with prior values of the related variables. Therefore, the forecast errors comprise statistics that were not completely removed by the univariate serial process. However, if it is properly extracted, it will improved forecast values and accuracy of the autoforecast process. By including these additional variables, it generates a linear polynomial function time series process called a transfer polynomial process in the form of inputs and outputs relationship.

\section{Transfer Function Models}

Transfer function processes use linear polynomial functions of the in the form of stochastic model as succinctly shows in in Figure 2. In order words, the input series dictate the results through a liner transfer function of output series. 


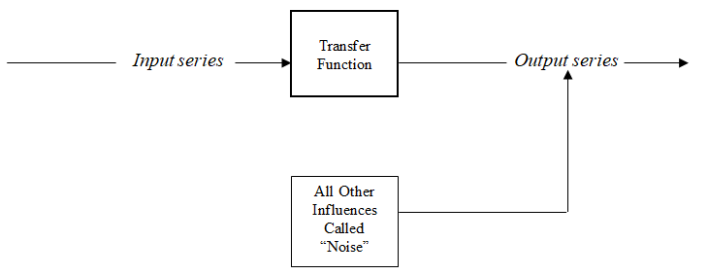

Figure 2. The Transfer Function Concept

Figure 3 illustrates the basic approach of a transfer function process and comprises of the following phases:

- Identification

- Parameters Estimation

- Diagnostic

- Checking

- Applicability of Transfer Function

Assume that data points for variables $x_{1 t}, x_{2 t}, \ldots$, such as price, exchange rates and an output variable $y_{t}$ for total quantity of wheat exported by the United States, then a stochastic features of the systems is govern by:

$$
\phi(B) y_{t}=\theta(B) x_{t} \text { or } y_{t}=\frac{\theta(B)}{\phi(B)} x_{t}
$$

Where

$$
\theta(B)=\left(\theta_{0}-\theta_{1} B-\ldots-\theta_{p} B^{p}\right) B^{p}
$$

and

$$
\phi(B)=\phi_{0}-\phi_{1} B-\ldots-\phi_{q} B^{q} .
$$

However, when there are more than a one inputs series then a more generalized ARIMA process such as:

$$
y_{t}=\sum_{i=1}^{k} \frac{\theta_{i}(B)}{\phi_{i}(B)} x_{i t}+\frac{\theta(B)}{\phi(B)} \alpha_{t}
$$

where $\alpha_{t}$ are independent, identical and normally distributed for series $a_{t}$ of the univariate process. In general $\theta_{i}(B) \neq \theta(B)$ and $\phi_{i}(B) \neq \phi(B)$. The procedures provide for model identification, diagnostic and fitting similar to Box-Jenkins for univariate models.

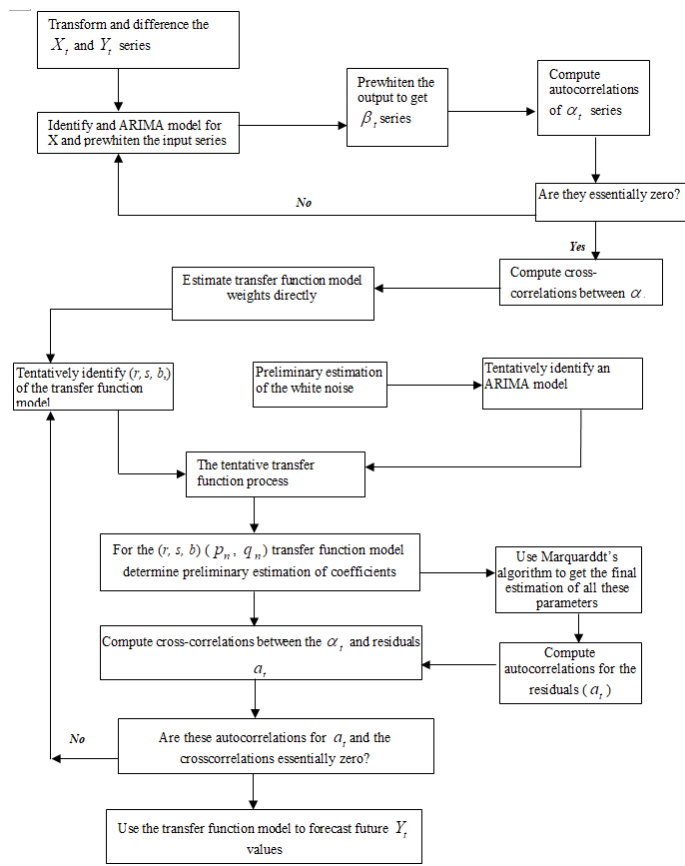

Figure 3. The Basic Steps in Developing a Transfer Function Model 


\section{Conclusions}

The forecast accuracy is determined quantitatively by the values of the obtained standard deviation of the residuals $\hat{\sigma}_{a}$. These values of $\hat{\sigma}_{a}$ for transfer function process and of the univariate model are evaluated. The forecast precision is determined by the mean square error (MSE). A time series process with least mean square error provides a more superior forecast than a process with high mean square error (Enders, 2003). It is the mean square of residuals of forecast values:

$$
\mathrm{MSE}=\frac{1}{n} \sum_{i=t_{0}}^{t_{0}+n}\left(Y_{i}-\hat{Y_{i}}\right)^{2}
$$

The MSE is calculated for $n+1$ forecasts from $t_{0}$ to $t_{0}+n$. For the forecast accuracy of the process, the $\sqrt{M S E}$ values for the forecasts for univariate and transfer function forecasting models are compared respectively. In addition, another criterion which has been advocated by Theil (1966) is to calculate the root mean square error of the predicted change, denoted:

$$
U=\sqrt{\frac{\frac{1}{n} \sum\left(\Delta Y_{i}-\Delta Y_{i}\right)^{2}}{\frac{1}{n} \sum\left(\Delta Y_{i}\right)^{2}}}
$$

This statistic has the advantage of possessing two natural calibration points. First, it is equal to 0 if the forecasts are perfectly accurate, and second, it is automatically equal to 1 for the naïve prediction of no change. If $\left(\Delta Y_{i}\right)$ is equal to 0 for each forecast, the numerator is equal to $\frac{1}{n} \sum\left(\Delta \hat{Y}_{i}-\Delta Y_{i}\right)^{2}$, which is the same as the denominator. Since a forecasting model ought to provide very minimum forecast variation, $U$ ought to falls between 0 and 1 , its closeness to 0 being an indicator of its relative success.

The results from this study provide valuable information about forecast framework and evaluation and modeling. First, the framework reveals that differencing stationary by unit-root tests using Augmented Dickey-Fuller procedure is needed before a transfer function can be applied to give a more accurate forecast values than the univariate. Second, several criteria are required to evaluate the accuracy of the transfer function process over the univariate process. The mean square error (MSE) values indicate that the transfer function model forecasts more accurately than the univariate model. The Theil value $(U)$ for the transfer function is lower than that of univariate process showing the superiority of the transfer function.

\section{References}

Anandalingan, G., \& Chen, L. (1989). Linear Combination of Forecasts: A General Bayesian Model. Journal of Forecasting, 8, 199-214. https://doi.org/10.1002/for.3980080306

Box, G. E. P., \& Jenkins, G. M. (1976). Time Series Analysis, Forecasting and Control (3rd ed.). Oakland, CA: Holden-Day.

Box, G. E. P., Jenkins, G. M., \& Rinsel, G. C. (1994). Time Series Analysis: Forecasting and Control. Engelwood Cliffs, NJ, Prentice-Hall.

Box, G. P., \& Jenkins, G. M. (1976). Time Series Analysis Forecasting and Control. San Francisco: Holden-Day.

Cleary, J. P., \& Levenbach, H. (1982). The Professional Forecaster: The Forecasting Process through Data Analysis. Belmont: Lifetime Learning Publications.

Clements, M. P., \& Hendry, D. F. (2002). Modelling methodology and forecast failure. Econometrics Journal, 5, 319-344. https://doi.org/10.1111/1368-423X.00086

Cooper, L. G., \& Nakanishi, M. (1988). Market Share Analysis: Evaluating Comparative Marketing Effectiveness. (New York, USA: Kluwer Academic). https://doi.org/10.1007/978-94-009-2681-3

Cooper, R. L. (1972). The Prediction Performance of Quarterly Econometric Models of the U.S. In Econometric Models of Cyclical Behavior. Ed. B. G. Hickman. New York: Columbia University Press, 1972.

Dalrymple, D. J. (1987). Sales Forecasting Practices: Results from a United States Survey. International Journal of Forecasting, 3, 379-91. https://doi.org/10.1016/0169-2070(87)90031-8 
Dave, C., \& DeJong, D. N. (2007). Structural Macroeconometrics. Princeton, New Jersey: Princeton University Press.

Dickey, D. A., Bell, W. R., \& Miller, R. B. (1986). Unit roots in time series models: Tests and implications. The American Statistician, 40(1), 12-26. https://doi.org/10.1080/00031305.1986.10475349

Diebold, F. X. (2007). Elements of Forecasting, (4th ed.). Mason, OH: Cengage Publishing.

Enders, W. (2008). Applied econometric time series. John Wiley \& Sons.

Gershenfeld, N. A., \& Weigend, A. S. (1994). The Future of Time Series: Learning and Understanding. In Weigend, Andreas S and Gershenfeld, N. A. (eds.), Time Series Prediction: Forecasting the Future and Understanding the Past. Reading, MA: Addison-Wesley.

Goodwin, B. K., \& Schroeder, T. C. (1991). Price dynamics in international wheat markets. Canadian Journal of Agricultural Economics/Revue canadienne d'agroeconomie, $237-254$. https://doi.org/10.1111/j.1744-7976.1991.tb03570.x

Granger, C. W. J. (2014). Forecasting in business and economics. Academic Press.

Granger, C. W. J., \& Newbold, P. (2014). Forecasting economic time series. Academic Press.

Labys, W. C. (1999). Forecasting Commodity Markets and Prices: Challenges and Possible Solutions. Published in the Proceedings of the Conference on the Dynamic Analysis and Forecasting of Commodity Markets and Prices (Dynamique des PriX et des marches de Matieres Premieres: Analyse et Prevision) GAMMAP, November 5-6, 1998, University of Grenoble.

Makridakis, S. (1986). The art and science of forecasting An assessment and future directions. International Journal of Forecasting, 2(1), 15-39. https://doi.org/10.1016/0169-2070(86)90028-2

Makridakis, S., Andersen, A., Carbone, R., Fildes, R., Hibon, M., Lewandowski, R., ... Winkler, R. (1982). The accuracy of extrapolation (time series) methods: Results of a forecasting competition. Journal of forecasting, 1(2), 111-153. https://doi.org/10.1002/for.3980010202

Mohanty, S., Meyers, W. H., \& Smith, D. B. (1999). A reexamination of price dynamics in the international wheat market. Canadian Journal of Agricultural Economics/Revue canadienne d'agroeconomie, 47(1), 21-29. https://doi.org/10.1111/j.1744-7976.1999.tb00213.x

Nelson, C. R., \& Plosser, C. R. (1982). Trends and random walks in macroeconmic time series: some evidence and implications. Journal of monetary economics, 10(2), 139-162. https://doi.org/10.1016/0304-3932(82)90012-5

Rudebusch, G. D. (1993). The uncertain unit root in real GNP. The American Economic Review, 83(1), $264-272$.

Theil, H. (1966). Applied Economic Forecasting. Amsterdam: North-Holland.

\section{Copyrights}

Copyright for this article is retained by the author(s), with first publication rights granted to the journal.

This is an open-access article distributed under the terms and conditions of the Creative Commons Attribution license (http://creativecommons.org/licenses/by/4.0/). 